JOURNAL OF INTEGRAL EQUATIONS

AND APPLICATIONS

Volume 15, Number 1, Spring 2003

\title{
THREE-POINT BOUNDARY VALUE PROBLEMS WITH SOLUTIONS THAT CHANGE SIGN
}

\author{
G. INFANTE AND J.R.L. WEBB
}

\begin{abstract}
Using the theory of fixed point index, we give new results for some three-point boundary value problems. In particular we study problems where the associated integral equation has a kernel that changes sign, so that positive solutions cannot exist. We obtain the existence of at least one or of multiple nonzero solutions.
\end{abstract}

1. Introduction. In this paper we study the existence of nonzero solutions of second order differential equations of the form

$$
u^{\prime \prime}(t)+g(t) f(u(t))=0, \quad 0<t<1
$$

under one of the boundary conditions (BC's)

$$
\begin{array}{ll}
u^{\prime}(0)=0, \alpha u(\eta)=u(1), & 0<\eta<1, \\
u(0)=0, \alpha u(\eta)=u(1), & 0<\eta<1 .
\end{array}
$$

These so-called three-point boundary value problems (BVP's), and more general $m$-point BVP's, are examples of nonlocal boundary conditions whose study has been motivated by work of Bitsadze and Samarskii and Il'in and Moiseev [6]. In recent years, the existence of solutions of equations more general than (1.1) has been thoroughly studied by Gupta, et al., see, for example, $[\mathbf{3}, \mathbf{4}]$ and the references therein.

Other authors, for example Ma [9] and Webb [11], have studied the existence of one or of multiple positive solutions when $0<\alpha<1$ for (1.2a) and $0<\alpha \eta<1$ for $(1.2 \mathrm{~b})$.

One approach is to write the BVP as an equivalent Hammerstein integral equation

$$
u(t)=\int_{0}^{1} k(t, s) g(s) f(u(s)) d s:=T u(t)
$$

2000 AMS Mathematics Subject Classification. Primary 34B10, Secondary 34B18, 47H10, 47H30.

Key words and phrases. Fixed point index, cone, nonzero solution.

Financial support by INdAM is gratefully acknowledged.

Copyright (C)2003 Rocky Mountain Mathematics Consortium 
and find a solution as a fixed point of the operator $T$ by using the classical theory of fixed point index in cones.

In the present paper we shall consider the other possible ranges for the parameter $\alpha$. For (1.2a) when $\alpha<0$, the kernel $k(t, s)$ is not positive for all values of $t, s$, indeed $k(1, s)<0$ for all $s$. Therefore, when $g$ and $f$ are positive, a fixed point of the operator $T$ cannot be positive on $[0,1]$.

Nevertheless, as we intend to show in this paper, it is possible to prove that nonzero solutions exist which have the property that they are positive (or negative) on some subinterval $[a, b]$ of $[0,1]$.

We shall show that one or more nonzero solutions exists for each of the other possible range of parameter $\alpha$ in each of the BC's above under suitable conditions on the nonlinear term.

While there are many publications dealing with the existence of multiple positive solutions, we believe our work establishing the existence of multiple nonzero solutions that change sign by using fixed point index theory is new.

In a recent paper, Lan and Webb $[\mathbf{8}]$ used the cone

$$
\tilde{K}=\{u \in C[0,1]: u \geq 0, \min \{u(t): a \leq t \leq b\} \geq c\|u\|\}
$$

(which is of a type due to Guo, see for example [5]) to prove that at least one positive solution existed for some boundary conditions of separated type. These results strictly included $f$ being either sublinear or superlinear. These results have been improved by Lan $[\mathbf{7}]$ to yield existence of multiple positive solutions under suitable conditions on $f$ for the separated BC's.

Using $\tilde{K}$, rather than $P=\{x \in C[0,1]: x(t) \geq 0$ for $t \in[0,1]\}$, has been purely a device to prove existence of positive solutions.

However, we employ the similar but larger cone

$$
K=\{u \in C[0,1]: \min \{u(t): a \leq t \leq b\} \geq c\|u\|\}
$$

and this seems to be natural for the type of nonzero solutions that we study.

The methods we use are rather similar to those of Lan [7] but we seek solutions of a different type. 
The conditions we impose on $g$ are quite weak, for example we can allow $g$ to be a non-negative $L^{1}$ function which is positive on a set of positive measure.

We suppose $f$ is positive; some of our other hypotheses involve

$$
\lim _{x \rightarrow 0+} f(x) / x \text { and } \lim _{x \rightarrow \infty} f(x) / x .
$$

Our conditions strictly include the sublinear and superlinear cases.

2. Existence of nonzero solutions of Hammerstein integral equations. We begin by giving some results for the following Hammerstein integral equation.

$$
u(t)=\int_{0}^{1} k(t, s) g(s) f(u(s)) d s \equiv T u(t) .
$$

We shall make the following assumptions on $f, g$ and the kernel $k$.

(F) $f: \mathbf{R} \rightarrow[0, \infty)$ is continuous.

(C) $k:[0,1] \times[0,1] \rightarrow \mathbf{R}$ is continuous and there exist a measurable function $\Phi:[0,1] \rightarrow[0, \infty)$, a subinterval $[a, b]$ and $c \in(0,1]$ such that

$$
|k(t, s)| \leq \Phi(s) \quad \text { for } t, s \in[0,1],
$$

and

$$
c \Phi(s) \leq k(t, s) \quad \text { for } t \in[a, b] \text { and } s \in[0,1]
$$

(G) $g:[0,1] \rightarrow[0, \infty)$ is measurable and $\int_{0}^{1} \Phi(s) g(s) d s<\infty$.

The hypothesis $(\mathrm{C})$ means finding upper bounds for $|k(t, \cdot)|$ when $t \in[0,1]$ and lower bounds of the same form for $k(t, \cdot)$ with $t \in[a, b]$. In applications we have some freedom of choice in determining $a, b$. We need $k(t, s)$ to be positive for all $t \in[a, b]$ and $s \in[0,1]$, but we do not require $k(t, s)$ to be positive for all $t, s$.

These hypotheses will allow us to work in the cone

$$
K=\{u \in C[0,1]: \min \{u(t): a \leq t \leq b\} \geq c\|u\|\} .
$$


This is a larger cone than the one used by Lan [7]. Note that functions in $K$ are positive on the subinterval $[a, b]$ but may change sign on $[0,1]$.

In order to use the well-known fixed point index for compact maps, we need to prove that $T: K \rightarrow K$ is compact, that is, $T$ is continuous and $\overline{T(Q)}$ is compact for each bounded subset $Q \subset K$.

Theorem 2.1. Assume that $(\mathrm{F}),(\mathrm{G})$ and $(\mathrm{C})$ hold. Then $T$ maps $K$ into $K$ and is compact.

Proof. Let $\mathcal{T}: C[0,1] \rightarrow C[0,1]$ be defined by $(\mathcal{T} x)(t)=$ $\int_{0}^{1} k(t, s) g(s) x(s) d s$. Then the kernel has the properties:

(i) $\int_{0}^{1}|k(t, s)| g(s) d s \leq \int_{0}^{1} \Phi(s) g(s) d s$ for all $t \in[0,1]$.

(ii) For each $\tau \in[0,1], \lim _{t \rightarrow \tau} \int_{0}^{1}|k(t, s) g(s)-k(\tau, s) g(s)| d s=0$.

To see (ii), note that if $t_{n} \rightarrow \tau$, then $\left|k\left(t_{n}, s\right) g(s)-k(\tau, s) g(s)\right| \rightarrow 0$ and

$$
\left|k\left(t_{n}, s\right) g(s)-k(\tau, s) g(s)\right| \leq 2 \Phi(s) g(s) \quad \text { for every } n .
$$

Therefore, by the dominated convergence theorem, (ii) holds. Since $[0,1]$ is compact, the limit in (ii) is uniform in $\tau$. Hence Proposition 3.4 $[10$, p. 167] shows that $\mathcal{T}: C[0,1] \rightarrow C[0,1]$ is compact. As $f$ is continuous, it follows that $T: C[0,1] \rightarrow C[0,1]$ is compact.

Furthermore we see that $T: K \rightarrow K$. Indeed, we have

$$
|T u(t)| \leq \int_{0}^{1}|k(t, s)| g(s) f(u(s)) d s
$$

so that

$$
\|T u\| \leq \int_{0}^{1} \Phi(s) g(s) f(u(s)) d s .
$$

Also

$$
\min _{a \leq t \leq b}\{T u(t)\} \geq c \int_{0}^{1} \Phi(s) g(s) f(u(s)) d s .
$$

Hence $T u \in K$ for every $u \in K$. 
We require some knowledge of the classical fixed point index for compact maps, see for example [1] or [5] for further information.

Let $K$ be a cone in a Banach space $X$. If $\Omega$ is a bounded open subset of $K$ (in the relative topology) we denote by $\bar{\Omega}$ and $\partial \Omega$ the closure and the boundary relative to $K$. When $D$ is an open bounded subset of $X$ we write $D_{K}=D \cap K$, an open subset of $K$.

Lemma 2.2. Let $D$ be an open bounded set with $D_{K} \neq \varnothing$ and $\bar{D}_{K} \neq K$. Assume that $T: \bar{D}_{K} \rightarrow K$ is a compact map such that $x \neq T x$ for $x \in \partial D_{K}$. Then the fixed point index $i_{K}\left(T, D_{K}\right)$ has the following properties.

(1) If there exists $e \in K \backslash\{0\}$ such that $x \neq T x+\lambda$ for all $x \in \partial D_{K}$ and all $\lambda>0$, then $i_{K}\left(T, D_{K}\right)=0$.

(2) If $\|T x\| \leq\|x\|$ for $x \in \partial D_{K}$, then $i_{K}\left(T, D_{K}\right)=1$.

(3) Let $D^{1}$ be open in $X$ with $\overline{D^{1}} \subset D_{K}$. If $i_{K}\left(T, D_{K}\right)=1$ and $i_{K}\left(T, D_{K}^{1}\right)=0$, then $T$ has a fixed point in $D_{K} \backslash \overline{D_{K}^{1}}$. The same result holds if $i_{K}\left(T, D_{K}\right)=0$ and $i_{K}\left(T, D_{K}^{1}\right)=1$.

Let $q: C[0,1] \rightarrow \mathbf{R}$ denote the continuous function $q(u)=\min \{u(t)$ : $a \leq t \leq b\}$. For $\rho>0$ we let $K_{\rho}:=\{x \in K:\|x\|<\rho\}$ and following Lan [7] we will use the set $\Omega_{\rho}:=\{u \in K: q(u)<c \rho\}$.

Lemma 2.3. $\Omega_{\rho}$ defined above has the following properties.

(a) $\Omega_{\rho}$ is open relative to $K$.

(b) $K_{c \rho} \subset \Omega_{\rho} \subset K_{\rho}$.

(c) $u \in \partial \Omega_{\rho}$ if and only if $q(u)=c \rho$.

(d) If $u \in \partial \Omega_{\rho}$, then $c \rho \leq u(t) \leq \rho$ for $t \in[a, b]$.

We omit the simple proof as it is exactly similar to the one in [7]. 
Notation. Let

$$
\begin{gathered}
f_{c \rho, \rho}=\min \{f(u) / \rho: u \in[c \rho, \rho]\}, \\
f^{-\rho, \rho}=\max \{f(u) / \rho: u \in[-\rho, \rho]\}, \\
M=\left(\min _{a \leq t \leq b} \int_{a}^{b} k(t, s) g(s) d s\right)^{-1}
\end{gathered}
$$

and

$$
m=\left(\max _{0 \leq t \leq 1} \int_{0}^{1}|k(t, s)| g(s) d s\right)^{-1} .
$$

We now prove two lemmas which give conditions when the fixed point index is either 0 or 1 .

Lemma 2.4. Suppose $\int_{a}^{b} \Phi(s) g(s) d s>0$ and that

$$
f_{c \rho, \rho} \geq M c \quad \text { and } \quad x \neq T x \quad \text { for } x \in \partial \Omega_{\rho} .
$$

Then $i_{K}\left(T, \Omega_{\rho}\right)=0$.

Proof. Let $e(t) \equiv 1$ for $t \in[0,1]$. Then $e \in K$. We prove that

$$
x \neq T x+\lambda e \quad \text { for } x \in \partial \Omega_{\rho} \quad \text { and } \lambda>0 .
$$

In fact, if not, there exist $x \in \partial \Omega_{\rho}$ and $\lambda>0$ such that $x=T x+\lambda e$. By condition $(*)$ and $(d)$ of Lemma 2.3, we have for $t \in[a, b]$,

$$
\begin{aligned}
x(t)= & \int_{0}^{1} k(t, s) g(s) f(x(s)) d s+\lambda \geq \int_{a}^{b} k(t, s) g(s) f(x(s)) d s+\lambda \\
& \geq c M \rho \int_{a}^{b} k(t, s) g(s) d s+\lambda \geq c \rho+\lambda .
\end{aligned}
$$

This implies that $q(x) \geq c \rho+\lambda>c \rho$ contradicting (c) of Lemma 2.3. Hence (1) of Lemma 2.2 gives $i_{K}\left(T, \Omega_{\rho}\right)=0$.

Lemma 2.5. Suppose $\max _{0 \leq t \leq 1} \int_{0}^{1}|k(t, s)| g(s) d s>0$ and that $f$ satisfies

$$
f^{-\rho, \rho} \leq m \quad \text { and } \quad x \neq T x \quad \text { for } x \in \partial K_{\rho}
$$


Then $i_{K}\left(T, K_{\rho}\right)=1$.

Proof. By $\left.{ }^{* *}\right)$, for $u \in \partial K_{\rho}$ and $t \in[0,1]$, we have

$$
\begin{aligned}
|T u(t)| & =\left|\int_{0}^{1} k(t, s) g(s) f(u(s)) d s\right| \leq \int_{0}^{1}|k(t, s)| g(s) f(u(s)) d s \\
& \leq m \rho \int_{0}^{1}|k(t, s)| g(s) d s \leq \rho=\|u\| .
\end{aligned}
$$

Therefore $\|T u\| \leq\|u\|$ for $u \in \partial K_{\rho}$. By (2) of Lemma 2.2, we have $i_{K}\left(T, K_{\rho}\right)=1$.

We now give our new result which asserts that Equation (2.1) has at least two nonzero solutions which are positive on the subinterval $[a, b]$.

Theorem 2.6. Assume that $\int_{a}^{b} \Phi(s) g(s) d s>0$ and one of the following conditions holds:

$\left(\mathrm{S}_{1}\right)$ There exist $\rho_{1}, \rho_{2}, \rho_{3} \in(0, \infty)$ with $\rho_{1}<c \rho_{2}$ and $\rho_{2}<\rho_{3}$ such that

$$
\begin{gathered}
f^{-\rho_{1}, \rho_{1}} \leq m, \quad f_{c \rho_{2}, \rho_{2}} \geq M c \\
x \neq T x \quad \text { for } x \in \partial \Omega_{\rho_{2}}, \quad \text { and } \quad f^{-\rho_{3}, \rho_{3}} \leq m .
\end{gathered}
$$

$\left(\mathrm{S}_{2}\right)$ There exist $\rho_{1}, \rho_{2}, \rho_{3} \in(0, \infty)$ with $\rho_{1}<\rho_{2}<c \rho_{3}$ such that

$$
\begin{aligned}
f_{c \rho_{1}, \rho_{1}} & \geq M c, \quad f^{-\rho_{2}, \rho_{2}} \leq m, \\
x \neq T x \quad \text { for } x & \in \partial K_{\rho_{2}}, \quad \text { and } \quad f_{c \rho_{3}, \rho_{3}} \geq M c .
\end{aligned}
$$

Then Equation (2.1) has two solutions in $K$ each of which is positive on $[a, b]$. Moreover, if in $\left(S_{1}\right), f^{-\rho_{1}, \rho_{1}} \leq m$ is replaced by $f^{-\rho_{1}, \rho_{1}}<m$, then Equation (2.1) has a third solution $x_{0} \in K_{\rho_{1}}$.

Proof. Assume that $\left(\mathrm{S}_{1}\right)$ holds. We show that either $T$ has a fixed point $x_{1}$ in $\partial K_{\rho_{1}}$ or in $\Omega_{\rho_{2}} \backslash \bar{K}_{\rho_{1}}$. If $x \neq T x$ for $x \in \partial K_{\rho_{1}} \cup \partial K_{\rho_{3}}$, by Lemmas 2.4 and 2.5, we have $i_{K}\left(T, K_{\rho_{1}}\right)=1, i_{K}\left(T, \Omega_{\rho_{2}}\right)=0$ and $i_{K}\left(T, K_{\rho_{3}}\right)=1$. By (b) of Lemma 2.3, we have $\bar{K}_{\rho_{1}} \subset K_{c \rho_{2}} \subset \Omega_{\rho_{2}}$ 
since $\rho_{1}<c \rho_{2}$. It follows from (3) of Lemma 2.2 that $T$ has a fixed point $x_{1}$ in $\Omega_{\rho_{2}} \backslash \bar{K}_{\rho_{1}}$. Similarly, $T$ has a fixed point $x_{2}$ in $K_{\rho_{3}} \backslash \bar{\Omega}_{\rho_{2}}$. The proof is similar when $\left(\mathrm{S}_{2}\right)$ holds.

Remark 2.7. Note that the third solution $x_{0} \in K_{\rho_{1}}$ might be zero. The other solutions are not because their norms are bounded away from zero. Although the statement and proof is almost identical to the similar result in [7] which deals with positive solutions, our new result allows solutions that are only positive on a subinterval and may change sign, and indeed this happens in the differential equations we consider below.

Theorem 2.6 can be generalized so as to assert the existence of many solutions, similar to a result of $[7]$. We could state such results for the existence of many solutions below but we give only the simpler results, leaving such statements to the reader.

Notation. Let

$$
\begin{aligned}
f^{0} & =\limsup _{u \rightarrow 0} \frac{f(u)}{|u|}, \quad f_{0}=\liminf _{u \rightarrow 0} \frac{f(u)}{|u|}, \\
f^{\infty} & =\limsup _{u \rightarrow \infty} \frac{f(u)}{u} \quad \text { and } \quad f_{\infty}=\liminf _{u \rightarrow \infty} \frac{f(u)}{u} .
\end{aligned}
$$

As a special case of Theorem 2.6 we have the following result.

Corollary 2.8. Assume that $\int_{a}^{b} \Phi(s) g(s) d s>0$ and there exists $\rho>0$ such that one of the following conditions holds.

$\left(\mathrm{E}_{1}\right) 0 \leq f^{0}<m, f_{c \rho, \rho} \geq M c, x \neq T x$ for $x \in \partial \Omega_{\rho}$, and $0 \leq f^{\infty}<m$.

$\left(\mathrm{E}_{2}\right) M<f_{0} \leq \infty, f^{-\rho, \rho} \leq m, x \neq T x$ for $x \in \partial K_{\rho}$, and $M<f_{\infty} \leq \infty$.

Then Equation (2.1) has two nonzero solutions in $K$.

Proof. We show that $\left(\mathrm{E}_{1}\right)$ implies $\left(\mathrm{S}_{1}\right)$. In fact, $0 \leq f^{0}<m$ implies that there exists $\rho_{1} \in(0, c \rho)$ such that $f^{-\rho_{1}, \rho_{1}}<m$. Let $\tau \in\left(f^{\infty}, m\right)$. Then there exists $r>\rho$ such that $f(u) \leq \tau u$ for $u \in[r, \infty)$ since $0 \leq f^{\infty}<m$. 
Let $\beta=\max \{f(u): 0 \leq u \leq r\}$ and $\rho_{3}>\beta /(m-\tau)$. Then we have

$$
f(u) \leq \tau u+\beta \leq \tau \rho_{3}+\beta<m \rho_{3} \quad \text { for } u \in\left[0, \rho_{3}\right] .
$$

This implies $f^{-\rho_{3}, \rho_{3}}<m$, hence $\left(\mathrm{S}_{1}\right)$ holds. Similarly, $\left(\mathrm{E}_{2}\right)$ implies $\left(\mathrm{S}_{2}\right)$.

By a similar argument to that of Theorem 2.6, we obtain the following new results on existence of at least one nonzero solution of Equation $(2.1)$.

Theorem 2.9. Assume that $\int_{a}^{b} \Phi(s) g(s) d s>0$ and one of the following conditions holds.

$\left(\mathrm{H}_{1}\right)$ There exist $\rho_{1}, \rho_{2} \in(0, \infty)$ with $\rho_{1}<c \rho_{2}$ such that

$$
f^{-\rho_{1}, \rho_{1}} \leq m \quad \text { and } \quad f_{c \rho_{2}, \rho_{2}} \geq M c .
$$

$\left(\mathrm{H}_{2}\right)$ There exist $\rho_{1}, \rho_{2} \in(0, \infty)$ with $\rho_{1}<\rho_{2}$ such that

$$
f_{c \rho_{1}, \rho_{1}} \geq M c \quad \text { and } \quad f^{-\rho_{2}, \rho_{2}} \leq m .
$$

Then Equation (2.1) has a nonzero solution in $K$.

Theorem 2.9 generalizes Theorem 2.2 in [ 8$]$ by allowing solutions that change sign.

Remark 2.10. We shall see below that, for certain values of the parameter $\alpha$, the kernel $k(t, s)$ is negative for $t$ in some interval $[a, b]$, for all $s$. In this case, assuming $g$ and $f$ are positive, we can show that nonzero solutions exist that are negative on $[a, b]$. Indeed, $u$ is a solution of

$$
u(t)=\int_{0}^{1} k(t, s) g(s) f(u(s)) d s
$$

if and only if $v:=-u$ is a solution of

$$
v(t)=\int_{0}^{1} \tilde{k}(t, s) g(s) \tilde{f}(v(s)) d s \equiv \tilde{T} v(t)
$$


where $\tilde{k}=-k$ and $\tilde{f}(v)=f(-v)$. Moreover $v$ is positive on $[a, b]$ if and only if $u$ is negative on $[a, b]$. Hence we can obtain results, exactly similar to the ones above, for the existence of solutions that are negative on $[a, b]$. We do not state the obvious theorems thus obtained.

3. Multiple nonzero solutions of problem $(\mathbf{1 . 2 a})$. We investigate the BVP

$$
u^{\prime \prime}+g(t) f(u)=0, \quad \text { a.e. on }[0,1]
$$

with boundary conditions

$$
u^{\prime}(0)=0, \quad \alpha u(\eta)=u(1), \quad 0<\eta<1 .
$$

By a solution of this BVP we will mean a solution of the corresponding Hammerstein integral equation

$$
u(t)=\int_{0}^{1} k(t, s) g(s) f(u(s)) d s .
$$

The solution of $u^{\prime \prime}+y=0$ with the BC's (3.2) is (by routine integration)

$u(t)=\frac{1}{1-\alpha} \int_{0}^{1}(1-s) y(s) d s-\frac{\alpha}{1-\alpha} \int_{0}^{\eta}(\eta-s) y(s) d s-\int_{0}^{t}(t-s) y(s) d s$

Thus the kernel [Green's function] of (3.3) is

$$
k(t, s)=\frac{1}{1-\alpha}(1-s)-\left\{\begin{array}{ll}
\frac{\alpha}{1-\alpha}(\eta-s) & s \leq \eta \\
0 & s>\eta
\end{array}- \begin{cases}t-s & s \leq t \\
0 & s>t .\end{cases}\right.
$$

We shall study separately the cases $\alpha<0$ and $\alpha>1$. The existence of positive solutions when $\alpha=0$ has been studied in [8], and when $0<\alpha<1$ in [11]. $\alpha=1$ is the resonance case and cannot be dealt with by the methods here, but existence in this case was studied in [2].

3.1 The case $\alpha<0$. To simplify the calculations we write $-\beta$ in place of $\alpha$, so that $\beta>0$. 
We have to exhibit $\Phi(s)$, a subinterval $[a, b] \subset[0,1]$ and a constant $c<1$ such that

$$
\begin{aligned}
|k(t, s)| & \leq \Phi(s) \quad \text { for every } t, s \in[0,1], \\
k(t, s) \geq c \Phi(s) & \text { for every } s \in[0,1], t \in[a, b] .
\end{aligned}
$$

We show that for these BC's we can take $\Phi(s)=(1-s)$.

\section{Upper bounds.}

Case 1. $s \leq \eta$. If $s>t$ then $k(t, s) \geq 0$ and

$$
k(t, s)=\frac{1-s}{1+\beta}+\frac{\beta}{1+\beta}(\eta-s) \leq \frac{1-s+\beta(1-s)}{1+\beta}=(1-s) .
$$

If $s \leq t$ then

$$
k(t, s)=\frac{1-s}{1+\beta}+\frac{\beta}{1+\beta}(\eta-s)-(t-s)=\frac{1+\beta \eta-t(1+\beta)}{1+\beta} .
$$

If $t \leq(1+\beta \eta) /(1+\beta)$ then $k(t, s) \geq 0$ and

$$
k(t, s)=\frac{1+\beta \eta-t(1+\beta)}{1+\beta} \leq \frac{1+\beta \eta-s(1+\beta)}{1+\beta} \leq(1-s) .
$$

If $t>(1+\beta \eta) /(1+\beta)$ then $k(t, s) \leq 0$ and

$$
\begin{aligned}
-k(t, s) & =\frac{-1-\beta \eta+t(1+\beta)}{1+\beta} \leq \frac{-1-\beta \eta+(1+\beta)}{1+\beta} \\
& =\frac{\beta(1-\eta)}{1+\beta} \leq \frac{\beta(1-s)}{1+\beta} .
\end{aligned}
$$

Case 2. $s>\eta$. If $s>t$ then

$$
k(t, s)=\frac{(1-s)}{1+\beta} \geq 0
$$


and we are done. If $s \leq t$ then

$$
k(t, s)=\frac{1+\beta s-t(1+\beta)}{1+\beta} .
$$

If $t \leq(1+\beta s) /(1+\beta)$ then $k(t, s) \geq 0$ and

$$
k(t, s) \leq \frac{1+\beta s-s(1+\beta)}{1+\beta}=\frac{(1-s)}{1+\beta} .
$$

If $t>(1+\beta s) /(1+\beta)$ then $k(t, s) \leq 0$ and

$$
-k(t, s)=\frac{-1-\beta s+t(1+\beta)}{1+\beta} \leq \frac{-1-\beta s+(1+\beta)}{1+\beta}=\frac{\beta(1-s)}{1+\beta} .
$$

Lower bounds. We show that we may take arbitrary $[a, b] \subset[0, \eta]$

Case 1. $s \leq \eta$. If $s>t$ then

$$
k(t, s)=\frac{1-s}{1+\beta}+\frac{\beta}{1+\beta}(\eta-s) \geq \frac{(1-s)}{1+\beta} .
$$

If $s \leq t$, since $t \leq b \leq \eta$ we have

$$
k(t, s) \geq \frac{1-s}{1+\beta}+\frac{\beta}{1+\beta}(\eta-s)-(\eta-s)=\frac{1-\eta}{1+\beta} \geq \frac{1-\eta}{1+\beta}(1-s) .
$$

Case 2. $s>\eta$. If $s>t$ then $k(t, s)=(1-s) /(1+\beta)$ and we are done. Since we take $b \leq \eta$ the case $s \leq t$ does not occur.

The conclusion is that we may take $c=(1-\eta) /(1+\beta)$.

Theorem 3.1. Let $a, b \in[0, \eta]$ and suppose that $\int_{a}^{b} g(s) d s>0$. Let $c$ be as given above. Let $m, M$ be as defined previously. Then for $\alpha<0$ the BVP (3.1), (3.2) has at least one nonzero solution, positive on $[a, b]$, if either

$\left(\mathrm{h}_{1}\right) 0 \leq f^{0}<m$ and $M<f_{\infty} \leq \infty$ or 
$\left(\mathrm{h}_{2}\right) 0 \leq f^{\infty}<m$ and $M<f_{0} \leq \infty$, and has two nonzero solutions, positive on $[a, b]$, if there is $\rho>0$ such that either

$\left(\mathrm{E}_{1}\right) 0 \leq f^{0}<m, f_{c \rho, \rho} \geq c M, x \neq T x$ for $x \in \partial \Omega_{\rho}$, and $0 \leq f^{\infty}<m$, or

$\left(\mathrm{E}_{2}\right) M<f_{0} \leq \infty, f^{-\rho, \rho} \leq m, x \neq$ Tx for $x \in \partial K_{\rho}$, and $M<f_{\infty} \leq \infty$.

We give a simple example to illustrate the theorem.

Example 3.2. Set $g \equiv 1$ and $f \equiv 2$, in this case $f^{\infty}=0, f_{0}=\infty$. The solution of (3.1) with (3.2) is $u(t)=-t^{2}+\left(1+\beta \eta^{2}\right) /(1+\beta)$. This is a solution that is positive on an interval containing $(0, \eta]$ but negative at $t=1$.

3.2 The case $\alpha>1$. For these BC's the kernel $k$ is negative on an interval so we apply Remark 2.10 and consider $-k$ in place of $k$. Thus we have to find $\Phi$ such that $|k(t, s)| \leq \Phi(s)$ for every $t, s \in[0,1]$ and show that there exists $[a, b] \subset[0,1]$ and a constant $c$ such that $-k(t, s) \geq c \Phi(s)$ for every $s \in[0,1]$ and $t \in[a, b]$. In fact we show that we can take

$$
\Phi(s)=\frac{\alpha}{\alpha-1}(1-s) .
$$

\section{Upper bounds.}

Case 1. $s \leq \eta$. If $s>t$ then

$$
-k(t, s)=\frac{1-s}{\alpha-1}-\frac{\alpha}{\alpha-1}(\eta-s)=\frac{1-s-\alpha \eta+\alpha s}{\alpha-1} .
$$

If $s \geq(-1+\alpha \eta) /(\alpha-1)$ (this occurs in particular when $\alpha \eta<1$ ) $-k(t, s) \geq 0$ and

$$
-k(t, s) \leq \frac{1-s-\alpha \eta+\alpha \eta}{\alpha-1}=\frac{(1-s)}{\alpha-1} .
$$

If $s<(-1+\alpha \eta) /(\alpha-1)$ then $k(t, s)>0$ and

$$
k(t, s)=\frac{-1+s+\alpha \eta-\alpha s}{\alpha-1} \leq \frac{-1+s+\alpha-\alpha s}{\alpha-1}=(1-s) .
$$


If $s \leq t$ then

$$
-k(t, s)=\frac{1-s}{\alpha-1}-\frac{\alpha}{\alpha-1}(\eta-s)+(t-s)=\frac{1-\alpha \eta+\alpha t-t}{\alpha-1} .
$$

If $t \geq(-1+\alpha \eta) /(\alpha-1)$ then $-k(t, s) \geq 0$ and

$$
-k(t, s) \leq \frac{1-\alpha \eta+\alpha-1}{\alpha-1} \leq \frac{\alpha(1-s)}{\alpha-1} .
$$

If $t<(-1+\alpha \eta) /(\alpha-1)$ then $k(t, s)>0$ and

$$
k(t, s)=\frac{-1+\alpha \eta-\alpha t+t}{\alpha-1} \leq \frac{-1+\alpha-\alpha s+s}{\alpha-1}=(1-s)
$$

Case 2. $s>\eta$. If $s>t$ then

$$
0 \leq-k(t, s)=\frac{(1-s)}{\alpha-1}
$$

and we are done.

If $s \leq t$ then

$$
0 \leq-k(t, s)=\frac{(1-s)}{\alpha-1}+(t-s) \leq \frac{\alpha(1-s)}{\alpha-1} .
$$

Lower bounds. We will show that we may take $a=\eta$ and $b \in(\eta, 1]$ which will yield a solution that is negative on $[\eta, b]$. But, if also $\alpha \eta<1$, we may take an arbitrary $[a, b] \subset[0,1]$. In particular this means that there exists a solution which is negative on the whole interval $[0,1]$ when $\alpha>1$ and $\alpha \eta<1$.

Case 1. $s \leq \eta$. If $s>t$ then

$$
-k(t, s)=\frac{1-s-\alpha \eta+\alpha s}{\alpha-1} \geq \frac{1-s-\alpha \eta+\alpha \eta s}{\alpha-1}=(1-\alpha \eta) \frac{(1-s)}{\alpha-1} .
$$

If $\alpha \eta<1$ we may take an arbitrary $a$, but if $\alpha \eta>1$ we would have a problem. However, if $\alpha \eta>1$ we choose $a \geq \eta$ so that this case does not occur. 
If $s \leq t$ and $\alpha \eta<1$ then

$$
-k(t, s) \geq \frac{1-\alpha \eta+\alpha s-s}{\alpha-1} \geq(1-\alpha \eta) \frac{(1-s)}{\alpha-1} .
$$

If $s \leq t$ and $\alpha \eta>1$, as we choose $a \geq \eta$ we have

$$
-k(t, s)=\frac{1-\alpha \eta+\alpha t-t}{\alpha-1} \geq \frac{1-\alpha \eta+\alpha \eta-\eta}{\alpha-1} \geq(1-\eta) \frac{(1-s)}{\alpha-1} .
$$

Case 2. $s>\eta$. If $s>t$ then

$$
-k(t, s)=\frac{(1-s)}{\alpha-1}
$$

and we are done. If $s \leq t$ then

$$
-k(t, s)=\frac{1+\alpha t-t-\alpha s}{\alpha-1} \geq \frac{1+\alpha s-s-\alpha s}{\alpha-1}=\frac{(1-s)}{\alpha-1} .
$$

The conclusion is that we may take either $a=\eta, b \in(\eta, 1]$ and $c=(1-\eta) / \alpha$ or, when $\alpha \eta<1$, we may take $a, b$ arbitrary and $c=(1-\alpha \eta) / \alpha$. Thus we can state the following results:

Theorem 3.3. Let $\alpha>1$, let $a=\eta, b \in(\eta, 1]$ (or $a, b$ arbitrarily chosen in $[0,1]$ if $\alpha \eta<1)$. Suppose that $\int_{a}^{b} g(s) d s>0$. Let $c$ be as given above and let $m, M$ be as defined previously. Then the BVP (3.1), (3.2) has at least one nonzero solution, negative on $[a, b]$, if either

$\left(\mathrm{h}_{1}\right) 0 \leq f^{0}<m$ and $M<f_{\infty} \leq \infty$ or

$\left(\mathrm{h}_{2}\right) 0 \leq f^{\infty}<m$ and $M<f_{0} \leq \infty$, and has two nonzero solutions, negative on $[a, b]$, if there is $\rho>0$ such that either

$\left(\mathrm{E}_{1}\right) 0 \leq f^{0}<m, f_{c \rho, \rho} \geq c M, x \neq T x$ for $x \in \partial \Omega_{\rho}$ and $0 \leq f^{\infty}<m$, or

$\left(\mathrm{E}_{2}\right) M<f_{0} \leq \infty, f^{-\rho, \rho} \leq m, x \neq T x$ for $x \in \partial K_{\rho}$ and $M<f_{\infty} \leq$ $\infty$.

We illustrate the theorem with the following simple example. 
Example 3.4. Let $\alpha>1$, and take $g \equiv 1, f \equiv 2$. The solution of the BVP (3.1), (3.2) is $u(t)=\left(\alpha \eta^{2}-1\right) /(\alpha-1)-t^{2}$. If $\alpha \eta<1$ this solution is negative on all of $[0,1]$. When $\alpha \eta^{2}>1$ the solution is negative on an interval, for example, when $\eta=1 / 2$ and $\alpha=5$ the solution is $u(t)=1 / 16-t^{2}$. By taking $\alpha$ very large, the interval on which the solution is negative approaches $(\eta, 1]$, hence our choice of $[a, b]$ is optimal in giving the largest interval on which the solution is negative.

4. Multiple nonzero solutions of problem (1.2b). We now investigate the second BVP

$$
u^{\prime \prime}+g(t) f(u)=0, \quad \text { a.e on }[0,1],
$$

with boundary conditions

$$
u(0)=0, \alpha u(\eta)=u(1), 0<\eta<1 .
$$

The kernel in this case is

$$
k(t, s)=\frac{1}{1-\alpha \eta} t(1-s)-\left\{\begin{array}{ll}
\frac{\alpha t}{1-\alpha \eta}(\eta-s) & s \leq \eta \\
0 & s>\eta
\end{array}- \begin{cases}t-s & s \leq t \\
0 & s>t .\end{cases}\right.
$$

4.1 The case $\alpha \eta<0$. Again we write $\beta=-\alpha>0$. We show that we may take

$$
\Phi(s)=(1+\beta) \frac{s(1-s)}{1+\beta \eta} .
$$

\section{Upper bounds.}

Case 1. $s \leq \eta$. If $s>t$ then $k(t, s) \geq 0$ and

$k(t, s)=\frac{t(1-s)+\beta t(\eta-s)}{1+\beta \eta} \leq \frac{s(1-s)+\beta s(1-s)}{1+\beta \eta}=(1+\beta) \frac{s(1-s)}{1+\beta \eta}$.

If $s \leq t$,

$$
k(t, s)=\frac{t(1-s)}{1+\beta \eta}+\frac{\beta t}{1+\beta \eta}(\eta-s)-(t-s)=\frac{-s t-\beta t s+s+\beta \eta s}{1+\beta \eta},
$$


and $k(s, t)$ is negative for $t>(1+\beta \eta) /(1+\beta)$ and $s \neq 0$; note also that $(1+\beta \eta) /(1+\beta)>\eta$.

For $k(t, s) \geq 0$ we have

$$
\begin{aligned}
k(t, s) & =\frac{-s t-\beta t s+s+\beta \eta s}{1+\beta \eta} \leq \frac{s(1-s)+\beta s(\eta-s)}{1+\beta \eta} \\
& \leq(1+\beta) \frac{s(1-s)}{1+\beta \eta}
\end{aligned}
$$

and for $k(t, s)<0$ we have

$$
-k(t, s)=\frac{s t+\beta t s-s-\beta \eta s}{1+\beta \eta} \leq \frac{\beta s(1-\eta)}{1+\beta \eta} \leq \beta \frac{s(1-s)}{1+\beta \eta} .
$$

Case 2. $s>\eta$. If $s>t$ then $k(t, s) \geq 0$

$$
k(t, s)=\frac{t(1-s)}{1+\beta \eta} \leq \frac{s(1-s)}{1+\beta \eta} .
$$

If $s \leq t$ then

$$
k(t, s)=\frac{-t s-\beta \eta t+s+\beta \eta s}{1+\beta \eta}
$$

and $k(s, t)$ is negative for $t>(s(1+\beta \eta)) /(s+\beta \eta)$. When $k(t, s) \geq 0$ we have

$$
k(t, s)=\frac{-t s-\beta \eta t+s+\beta \eta s}{1+\beta \eta} \leq \frac{-s^{2}-\beta \eta s+s+\beta \eta s}{1+\beta \eta}=\frac{s(1-s)}{1+\beta \eta},
$$

and when $k(t, s)<0$ we have

$$
-k(t, s)=\frac{t s+\beta \eta t-s-\beta \eta s}{1+\beta \eta} \leq \frac{\beta \eta(1-s)}{1+\beta \eta} \leq \beta \frac{s(1-s)}{1+\beta \eta} .
$$

Lower bounds. We show that we may take arbitrary $[a, b] \subset(0, \eta]$.

Case 1. $s \leq \eta$. If $s>t$ then

$$
k(t, s)=\frac{t-s t+\beta t \eta-\beta t s}{1+\beta \eta} \geq \frac{t(1-s)}{1+\beta \eta} \geq \frac{a(1-s)}{1+\beta \eta} \geq a \frac{s(1-s)}{1+\beta \eta} .
$$


If $s \leq t$ and $t \leq b \leq \eta$,

$$
k(t, s) \geq \frac{-s \eta-\beta \eta s+s+\beta \eta s}{1+\beta \eta}=\frac{s(1-\eta)}{1+\beta \eta} \geq(1-\eta) \frac{s(1-s)}{1+\beta \eta} .
$$

Case 2. $s>\eta$. If $s>t$ then

$$
k(t, s)=\frac{t(1-s)}{1+\beta \eta} \geq \frac{a(1-s)}{1+\beta \eta} \geq a \frac{s(1-s)}{1+\beta \eta} .
$$

The case $s \leq t$ does not occur since we take $b \leq \eta$. Therefore we may take

$$
c=\min \{a, 1-\eta\} /(1+\beta) .
$$

Now it is clear that a theorem exactly similar to Theorem 3.1 holds. We leave the obvious statement to the reader. The following simple example illustrates the unstated result.

Example 4.1. Set $g \equiv 1$ and $f \equiv 2$. The solution of (4.1) with (4.2) is

$$
u(t)=\frac{1+\beta \eta^{2}}{1+\beta \eta} t-t^{2} .
$$

Thus $u(t)$ is positive on $[0, \eta]$ but $u(1)<0$.

4.2 The case $\alpha \eta>1$. For these BC's the kernel $k$ is negative on an interval so we apply Remark 2.10. We show that for these BC's we may take

$$
\Phi(s)=\alpha \frac{s(1-s)}{\alpha \eta-1} .
$$

\section{Upper bounds.}

Case $1 . s \leq \eta$. If $s>t$ then

$$
-k(t, s)=\frac{t(1-s-\alpha \eta+\alpha s)}{\alpha \eta-1} \leq \frac{t(1-s)}{\alpha \eta-1} \leq \frac{s(1-s)}{\alpha \eta-1} .
$$


Also

$k(t, s)=\frac{t(-1+s+\alpha \eta-\alpha s)}{\alpha \eta-1} \leq \frac{t(-1+s+\alpha-\alpha s)}{\alpha \eta-1} \leq(\alpha-1) \frac{s(1-s)}{\alpha \eta-1}$.

If $s \leq t$ then

$$
-k(t, s)=\frac{-s t+\alpha t s-\alpha \eta s+s}{\alpha \eta-1}=\frac{s(-t+\alpha t-\alpha \eta+1)}{\alpha \eta-1} .
$$

When $t \geq(\alpha \eta-1) /(\alpha-1)$ then $-k(s, t) \geq 0$ and

$$
-k(t, s) \leq \frac{s(\alpha-\alpha \eta)}{\alpha \eta-1} \leq \alpha \frac{s(1-s)}{\alpha \eta-1} .
$$

If $t<(\alpha \eta-1) /(\alpha-1)$ then $k(s, t) \geq 0$ and

$-k(t, s) \leq \frac{s(s-1+\alpha(\eta-s))}{\alpha \eta-1} \leq \frac{s(s-1+\alpha(1-s))}{\alpha \eta-1} \leq(\alpha-1) \frac{s(1-s)}{\alpha \eta-1}$.

Case 2. $s>\eta$. If $s>t$ then $-k(t, s) \geq 0$ and

$$
-k(t, s)=\frac{t(1-s)}{\alpha \eta-1} \leq \frac{s(1-s)}{\alpha \eta-1} .
$$

If $s \leq t$ then $-k(t, s) \geq 0$ and

$$
\begin{aligned}
-k(t, s) & =\frac{-t s+\alpha \eta t-\alpha \eta s+s}{\alpha \eta-1} \leq \frac{-s+\alpha \eta-\alpha \eta s+s}{\alpha \eta-1}=\frac{\alpha \eta(1-s)}{\alpha \eta-1} \\
& <\alpha \frac{s(1-s)}{\alpha \eta-1}
\end{aligned}
$$

Lower bounds. We show that we may take an arbitrary $[a, b] \subset$ $[\eta, 1]$.

Case 1. $s \leq \eta$. Since we take $a \geq \eta$ we only have the case $s \leq t$ and then

$$
-k(t, s)=\frac{s(-t+\alpha t-\alpha \eta+1)}{\alpha \eta-1} .
$$


Since $\eta>\alpha \eta-1 / \alpha-1$ we have $-k(t, s) \geq 0$ and

$$
-k(t, s) \geq \frac{s(-\eta+\alpha \eta-\alpha \eta+1)}{\alpha \eta-1} \geq(1-\eta) \frac{s(1-s)}{\alpha \eta-1} .
$$

Case 2. $s>\eta$. If $s>t$ then

$$
-k(t, s)=\frac{t(1-s)}{\alpha \eta-1} \geq \frac{a(1-s)}{\alpha \eta-1} \geq a \frac{s(1-s)}{\alpha \eta-1} .
$$

If $s \leq t$ then

$$
-k(t, s)=\frac{-t s+\alpha \eta t-\alpha \eta s+s}{\alpha \eta-1} \geq \frac{-s^{2}+\alpha \eta s-\alpha \eta s+s}{\alpha \eta-1}=\frac{s(1-s)}{\alpha \eta-1} .
$$

Thus we may take $c=\min \{a, 1-\eta\} / \alpha$. A theorem exactly similar to Theorem 3.3 holds. We omit its obvious statement.

\section{REFERENCES}

1. H. Amann, Fixed point equations and nonlinear eigenvalue problems in ordered Banach spaces, SIAM. Rev. 18 (1976), 620-709.

2. W. Feng and J.R.L. Webb, Solvability of three point boundary value problems at resonance, Nonlinear Anal. 30 (1997), 3227-3238.

3. C.P. Gupta, S.K. Ntouyas and P. CH. Tsamatos, On an m-point boundaryvalue problem for second-order ordinary differential equations, Nonlinear Anal. 23 (1994), 1427-1436 .

4. C.P. Gupta and S.I. Trofimchuk, Existence of a solution of a three-point boundary value problem and the spectral radius of a related linear operator, Nonlinear Anal. 34 (1998), 489-507.

5. D. Guo and V. Lakshmikantham, Nonlinear problems in abstract cones, Academic Press, New York, 1988.

6. V. Il'in and E. Moiseev, Nonlocal boundary value problems of the first kind for a Sturm-Liouville operator in its differential and finite difference aspects, Differential Equations 23 (1987), 803-810.

7. K.Q. Lan, Multiple positive solutions of semilinear differential equations with singularities, J. London Math. Soc. (2) 63 (2001), 690-704.

8. K.Q. Lan and J.R.L. Webb, Positive solutions of semilinear differential equations with singularities, J. Differential Equations 148 (1998), 407-421.

9. R. Ma, Positive solutions of a nonlinear three-point boundary-value problem, Electron. J. Differential Equations 34 (1999), 1-8. 
10. R.H. Martin, Nonlinear operators $\mathcal{E}$ differential equations in Banach spaces, Wiley, New York, 1976.

11. J.R.L. Webb, Positive solutions of some three point boundary value problems via fixed point index theory, Nonlinear Anal. 47 (2001), 4319-4332.

Dipartimento di Matematica, Università della Calabria, 87036 ArcavaCAta di Rende, Cosenza

E-mail address: infanteg@unical.it

Department of Mathematics, University of Glasgow, Glasgow G12 $8 \mathrm{QW}, \mathrm{UK}$

E-mail address: jrlw@maths.gla.ac.uk 